\title{
Argumentación y uso de pruebas: realización de inferencias sobre una secuencia de icnitas
}

\section{Argumentation and use of evidence: making inferences about a sequence of footprints}

\author{
Paloma Blanco Anaya, Joaquín Díaz de Bustamante \\ Departamento de Didáctica das Ciencias Experimentais. \\ Universidade de Santiago de Compostela \\ paloma.blanco@usc.es, joaquin.diaz@usc.es
}

RESUMEN • En el presente artículo se analiza el proceso de razonamiento de los estudiantes a través de la argumentación, para lo que se hace uso del modelo de argumentación de Toulmin (1958). La tarea propuesta contiene un conjunto de icnitas, cuyo propósito es esclarecer qué ocurrió. Los objetivos que guían este trabajo son dos: promover el razonamiento científico y analizar el discurso argumentativo de los estudiantes. Los resultados indican que esta actividad permite al alumnado, por un lado, establecer numerosas inferencias pese a la falta de información sobre la secuencia de huellas y, por otro lado, alcanzar un alto nivel de complejidad en sus argumentos, puesto que fueron capaces de formular tanto contraargumentos como refutaciones (Kuhn, 1991).

PALABRAS CLAVE: competencia científica; construcción del conocimiento; argumentación; inferencia; icnitas.

ABSTRACT - This article analyzes the reasoning process of students through argumentation, using the Toulmin's argument pattern (1958). The task includes a set of footprints, whose purpose is to clarify what happened. The objectives that guide this work are: to promote the scientific reasoning and to analyse the students' argumentative discourse. The results show that this task allowed to the students, on the one hand, to establish numerous inferences in spite of the lack of information about the sequence of footprints and, on the other hand, to achieve high level of complexity in their arguments because of they were able to formulate not only counterarguments but also rebuttals (Kuhn, 1991).

KEYWORDS: scientific competence; knowledge’s construction; argumentation; inference; footprints. 


\section{INTRODUCCIÓN}

El propósito de este artículo es analizar el proceso de razonamiento de los estudiantes a través de la argumentación, en términos de uso de datos y pruebas que les lleven a enunciar una conclusión. El contexto empleado corresponde a una situación problemática en la que los estudiantes deben interpretar un conjunto de marcas que simulan una secuencia compleja de huellas fósiles o icnitas (Lenderman y Abd-El-Khalick, 1998) para explicar el acontecimiento que las originó.

La relevancia de la propuesta dentro del contexto educativo actual es la de contribuir a la formación de los estudiantes desarrollando su competencia científica, lo que incluye la capacidad de "utilizar el conocimiento científico en contextos cotidianos, de aplicar los procesos que caracterizan a las ciencias y sus métodos de investigación" (Cañas, Martín-Díaz y Nieda, 2007, p. 33). Las directrices de PISA (OCDE, 2006) desglosan la competencia científica en tres dimensiones: "identificar cuestiones científicas, explicar fenómenos de forma científica y usar pruebas científicas”. En la actividad que se plantea, la primera dimensión está ya determinada por la propia tarea y, por tanto, se trabajan principalmente las dos últimas dimensiones. Así, la "explicación de fenómenos de forma científica" corresponde al hecho de dar una explicación, describiendo e interpretando lo que ocurrió en la secuencia de icnitas, y "el uso de pruebas científicas" es necesario para conferir consistencia a las afirmaciones, al emplearlas en sus argumentos y defenderlas ante sus compañeros. Entendemos que las tres dimensiones señaladas están interconectadas, como indican Jiménez Aleixandre, Bravo y Puig (2009), y todas ellas han de ser empleadas por los estudiantes en la resolución de problemas científicos.

En este contexto, nuestro primer objetivo es promover el razonamiento científico a través de los procesos de observación, realización de inferencias y emisión de hipótesis o, en su caso, establecimiento de conclusiones por parte de los estudiantes. El segundo es analizar el discurso argumentativo mediante el cual los estudiantes justifican sus conclusiones.

\section{CONSTRUCCIÓN DEL CONOCIMIENTO CIENTÍFICO EN EL AULA}

La construcción del conocimiento científico de disciplinas como la biología y la geología se ha basado en gran medida en las observaciones (Del Carmen, 2011) y en las inferencias que se hacen, como sucedió con las teorías sobre el origen de los seres vivos: mientras que para los creacionistas los fósiles constituían pruebas de las sucesivas creaciones (Jiménez Aleixandre, 1996), para Darwin estos eran pruebas evidentes de su evolución. Las mismas observaciones acerca de la formación de los orógenos fueron interpretadas por fijistas y movilistas de manera completamente diferente; mientras que para los fijistas los orógenos son consecuencia de una elevación inicial del terreno, para los movilistas estos se originan por movimientos horizontales de los continentes (Anguita y Moreno, 1978). El concepto de "anteojos conceptuales" de Kuhn (1962), según el cual todo fenómeno observable será interpretado en función de las ideas del observador, es relevante para la explicación de esta disparidad de interpretaciones. Sin embargo, una gran parte del profesorado de ciencias considera que solo existe una forma de estudiar la realidad y que los datos no tienen más que una interpretación posible (Driver, Newton y Osborne, 2000), transmitiendo esta visión a los estudiantes.

Para romper con la percepción de que la ciencia solo genera verdades absolutas, idea que subyace en una gran mayoría del alumnado, diversos autores han manifestado la importancia de que los estudiantes aprendan a razonar científicamente. Lederman y Abd-El-Khalick (1998) consideran que en el aula se fomenta esta perspectiva de una única teoría verdadera por el hecho de que el alumnado resuelve ejercicios de respuesta única, realizan prácticas de laboratorio tipo "receta de cocina", etc. En opinión de Driver, Newton y Osborne (2000) esta concepción de la ciencia se debe a que es explicada como un conjunto de principios irrefutables, en lugar de enseñarse como un proceso social que requiere la 
interpretación de datos, evaluación de enunciados científicos y otras prácticas habituales de la actividad científica. Chinn y Malhotra (2002) aseguran que si queremos impulsar el razonamiento científico, debemos llevar al aula actividades que promuevan auténtica indagación.

Además de promover el razonamiento científico en el aula, hay que tener en cuenta que los obstáculos epistemológicos con los que se encontró la ciencia a lo largo de su historia pueden ser obstáculos también en el aprendizaje del alumnado, principalmente ocasionados por las vivencias cotidianas (Bachelard, 1938-1974) y vinculados al contexto científico-tecnológico actual, como afirma Pedrinaci (2001). Por lo tanto, será interesante ayudarlos a salvar estos obstáculos, ofreciéndoles la posibilidad de usar datos y pruebas para que argumenten y mejoren su comprensión acerca de la ciencia, siempre con la ayuda del docente, quien facilitará su aprendizaje.

En la práctica no es factible enseñar todas las teorías o hipótesis que existen para explicar un mismo hecho; no obstante, sí sería interesante trabajar con las pruebas que apoyan la teoría más aceptada y analizar otras menos relevantes, para mostrar que no hay un único camino en el que la ciencia es construida. Se trataría, siguiendo a Duschl y Erduran (1996), de que los estudiantes establecieran qué información o datos son necesarios y qué importancia y significado pueden tener las regularidades o pautas identificables para resolver el problema propuesto, explicándolo en el marco teórico adecuado.

En este ambiente, la construcción del conocimiento debe ser regulada por el propio estudiante, en un proceso de metaaprendizaje (Sanmartí, 2002), tomando conciencia de la progresión de su conocimiento. Para conseguirlo, se puede trabajar con la resolución de problemas que requiera la aplicación de metodologías propias del trabajo científico, como es el caso de los problemas auténticos (Jiménez Aleixandre, 1998). Sin que ello implique que el alumnado reproduzca los descubrimientos de la comunidad científica, pero que los problemas planteados sí constituyan un nuevo interrogante para ellos (Díaz de Bustamante y Jiménez Aleixandre, 1999).

De acuerdo con esta perspectiva, los problemas auténticos muestran una amplia gama de soluciones posibles en las que el alumnado debe escoger la más apropiada. En este momento es cuando el estudiante necesita hacer uso de su capacidad argumentativa, que trataremos a continuación, ya que a través de los argumentos y de sus justificaciones deberá persuadir a sus compañeros para llegar entre todos a una conclusión.

\section{ARGUMENTACIÓN Y USO DE PRUEBAS}

La argumentación, entendida como la capacidad de evaluar los datos y las pruebas para generar una conclusión científica que difiera de una mera opinión (Jiménez Aleixandre, 2010), es inherente a la construcción del conocimiento científico. Según esta autora, hay varios contextos en los que se puede trabajar el uso de pruebas, entre ellos el de proponer a los estudiantes que elijan entre teorías o modelos para explicar un suceso. En nuestro caso, como ya se señaló, se trata de encontrar la mejor explicación acerca de qué ocurrió en la secuencia de huellas, en la cual hay poca información disponible que nos permita establecer con precisión una afirmación categórica, debiendo valorarse diferentes opciones.

En opinión de Jiménez Aleixandre (2011), en argumentación es importante la práctica de evaluación del conocimiento, que consiste en valorar las diferentes explicaciones que conducen a una hipótesis adecuada a las pruebas. Aunque para evaluar el conocimiento no sea requisito indispensable el trabajo en grupos, nosotros sí lo consideramos oportuno para lograr, en primer lugar, la co-elaboración de los argumentos, en los que, según Jiménez Aleixandre, predomina el intento de persuasión entre los componentes. En segundo lugar, en un debate argumentado, los estudiantes deben tener en cuenta los argumentos opuestos, que, siguiendo con la opinión de Jiménez Aleixandre (2010), constituyen parte del pensamiento crítico, formando a personas activas socialmente. 
Kuhn (2005) sostiene que los participantes en un discurso argumentativo necesitan habilidades cognitivas para juzgar y seleccionar la respuesta más efectiva, lo que favorece que surjan figuras argumentativas tales como la contraargumentación y la refutación. Así, según esta autora (1991), los contraargumentos son argumentos alternativos al propio y al del oponente, mientras que las refutaciones son críticas a las pruebas de un argumento. Estas figuras manifiestan la complejidad del discurso de los estudiantes, que en opinión de autores como Erduran, Simon y Osborne (2004) supone evaluar la calidad de la argumentación según la cantidad y complejidad de las refutaciones. Para estos autores, mientras que los contraargumentos no cuestionan el argumento principal de forma importante, las refutaciones ponen en duda las pruebas con las que se sostiene un argumento, por ello las consideran como una medida de la alta calidad de la argumentación.

El desarrollo de la argumentación en este estudio tiene en cuenta el modelo de argumentación de Toulmin (1958), que engloba, de modo general, los datos, las pruebas, los respaldos teóricos y las conclusiones que enuncian los estudiantes. Sin embargo, coincidimos con Kelly y Takao (2002) en que este modelo, por un lado, es apto para argumentos con pocos elementos y, por otro lado, resulta escaso para argumentos con diferentes niveles de complejidad, en los que se sucede una cadena de razonamientos. De modo que estos autores ampliaron el modelo para integrar, en un mismo patrón, varias conclusiones que dan consistencia a un argumento de mayor nivel, esto es, argumentos subsecuentes de un argumento principal, lo que Kelly y Takao denominaron niveles epistémicos en argumentación.

\section{LA PROPUESTA DIDÁCTICA}

La propuesta didáctica (anexo I) está compuesta por un enunciado que especifica el contexto del problema: "Un yacimiento paleontológico de Soria", sigue con la imagen de la secuencia de icnitas y termina indicando que, por grupos, hay que esclarecer qué ocurrió en esta secuencia. La fuente original de las pisadas es Hurd et al. (1989), de la que Lockley (1993) asegura que, pese a que pueda parecer una secuencia real para los no expertos, está inventada para proponerla como ejercicio.

En este punto queremos destacar que no se trata de un problema de geología en sí mismo, sino que su principal finalidad es la de trabajar la competencia científica y la argumentación, de forma conjunta.

La secuencia de icnitas empleadas en la propuesta estudiada en este trabajo ha sido utilizada por diversos autores con diferente finalidad. Según Lederman y Abd-El-Khalick (1998), esta actividad ayuda a que los estudiantes, por un lado, distingan entre observación e inferencia y, por otro, se den cuenta de que a partir del mismo conjunto de pruebas hay varias respuestas posibles. Izquierdo (2000) afirma que otra de las finalidades de esta propuesta es que los alumnos tengan constancia de que los científicos no saben lo que ocurrió realmente. En dicha propuesta se les enseña a los alumnos la secuencia de pisadas sin contexto, con lo que las posibles respuestas son altamente diversas. Sin embargo, en nuestro caso consideramos oportuno contextualizarla añadiendo el enunciado, antes mencionado, para propiciar el uso de pruebas, la argumentación y la contraargumentación entre los estudiantes.

Otros autores que basan una actividad en un conjunto de pisadas son Sequeiros, Pedrinaci y Berjillos (1996), pero en este caso la secuencia que proponen es diferente a la empleada en el presente estudio. Estos autores asignan el siguiente título a la actividad: “QQuién se comió al dinosaurio?”. Lo que se busca es que los estudiantes reconstruyan la secuencia de acontecimientos de un conjunto de icnitas más complejo que el de la propuesta de este trabajo por ser huellas más realistas, aunque con un grado de apertura menor. Como estos autores indican, en estas actividades no se intenta que los estudiantes realicen el trabajo de los científicos, pero esto no debe suponer un obstáculo para que resuelvan casos menos complejos empleando estas estrategias. 


\section{METODOLOGÍA}

\section{Participantes y obtención de los datos}

La actividad se llevó a cabo en un aula de primero de bachillerato, en "Ciencias para el mundo contemporáneo", formada por 23 estudiantes, 7 chicas y 16 chicos, con edades comprendidas entre 16 y 17 años.

Para resolver la actividad se dividió el aula en 6 grupos, a cada uno se le asignó una letra, desde la A hasta la F, y a cada participante se le atribuyó un pseudónimo que empezase por la letra del grupo al que pertenecía, respetando el género, a fin de mantener su anonimato. Disponían de 50 minutos, durante los cuales los estudiantes fueron grabados en audio y en vídeo, grabaciones que posteriormente se transcribieron para su análisis. Además, cada grupo entregó un pequeño informe escrito con la división en tramos de la secuencia de icnitas y con sus conclusiones.

\section{Análisis de los datos}

Para el análisis de la realización de la actividad se establecieron, previamente, como referencia, los criterios de resolución básicos. Se realizó un primer análisis, en el que se identificaron los tramos de la secuencia de huellas y se extrajo información de estas para, en un segundo análisis, inferir los sucesos que han tenido lugar en cada uno de los episodios. El hecho de haber elaborado el referencial nos ha permitido conocer que la planificación para resolver esta actividad podría comenzar por dividir la secuencia en tramos, con el fin de dar explicaciones parciales que luego concluyan con una afirmación global. Esta división referencial se puede observar en la figura 1 (R), que la hemos incluido en la misma figura, en la que se muestra la división realizada por los grupos, por considerarla más ilustrativa.

$\mathrm{El}$ argumento referencial que contienen las observaciones y respectivas inferencias se puede ver en el esquema 1. Para justificarlo, apoyamos nuestras interpretaciones en la paleoicnología. Según esta disciplina, el "ángulo de zancada" formado por tres pisadas consecutivas (con alternancia de extremidad) indica que a mayor ángulo, mayor velocidad, y viceversa (Lockley, 1993). A modo de resumen, una de las posibles interpretaciones de la secuencia de huellas es, en el primer tramo, que ambos individuos van andando; en el segundo, que el de huellas grandes empieza a correr, pues su ángulo de zancada aumenta, y en el tercero que los dos corren. En el cuarto, el ángulo de zancada no nos ayuda, pero en el quinto podemos volver a afirmar que las únicas pisadas que quedaron registradas indican que el individuo va andando. Por lo tanto, queda un último interrogante: ¿qué ha pasado con el individuo del que no quedan huellas registradas? Podría ser comido por el otro, salir volando, que sus huellas se borraran o incluso que las huellas de ambos individuos pertenezcan a momentos diferentes, es decir, que no fueran sucesos simultáneos. 


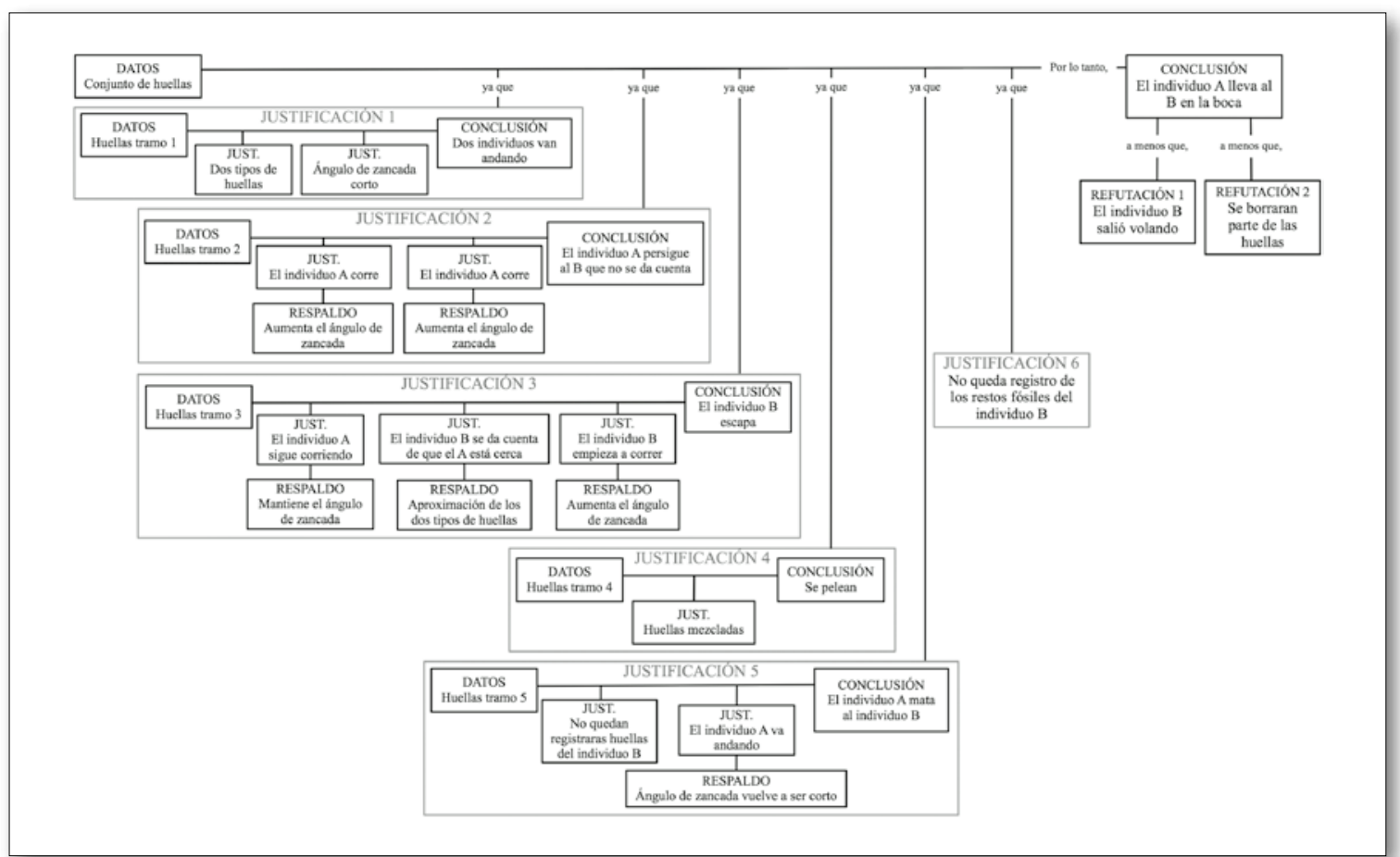

Esquema 1. Referencial

Al tratar este conjunto concreto de huellas, Lockley (1993) hace la siguiente interpretación: "Las huellas muestran a dos animales convergiendo. El depredador aumenta su velocidad y ataca al otro animal, la pobre víctima. Corren en círculos y la víctima sucumbe, quizás mareada. Entonces el depredador se marcha, probablemente con la víctima entre sus mandíbulas o en su estómago" (p. 242). Coincidimos con este autor en lo esencial de su interpretación, pero diferimos al considerar que es posible la existencia de otras soluciones alternativas.

Además de pedir una explicación de lo sucedido en la secuencia de icnitas, se les planteó si necesitaban más datos para corroborar sus afirmaciones. A este respecto, se esperaba que la información necesaria se refiriese, por ejemplo, a la profundidad de las huellas en diferentes tramos o a las dimensiones reales de ellas. Debido a esto, diferenciaremos las respuestas de los grupos en: conclusiones, si no dan respuesta a esta pregunta, o hipótesis, si indican parámetros que deben conocer para corroborarla.

El análisis del informe escrito, obtenido de cada uno de los grupos, consistió en comparar las divisiones de la secuencia de icnitas en tramos y obtener sus conclusiones finales.

En el caso de las transcripciones, estas se analizaron con el fin de conocer el razonamiento que siguen los estudiantes durante el discurso argumentativo. Para ello hemos empleado el modelo de argumentación de Toulmin (1958), que constituye una de las herramientas más empleadas para el análisis de argumentos, modificado por Kelly y Takao (2002), organizándolos como argumentos subsecuentes. Las diferencias entre el análisis de estos autores y nuestro estudio radican en que, en lugar de establecer niveles epistémicos, en este trabajo se categorizan los argumentos atendiendo a los tramos que dividen los acontecimientos de la secuencia de icnitas. En cuanto a las similitudes, estas radican en que se necesitan varias afirmaciones para apoyar el argumento principal. 


\section{RESULTADOS Y DISCUSIÓN}

Los resultados que se muestran a continuación se abordan con relación a los objetivos ya señalados. Se presentan en dos apartados con la idea de detallar, en el primero, el desarrollo de la actividad en los pequeños grupos, lo cual nos dará idea del proceso de razonamiento y, en el segundo, los argumentos empleados para dar respuesta al problema propuesto.

\section{Desarrollo de la actividad: observaciones e inferencias}

Los estudiantes dividieron la secuencia de icnitas en tramos, como se muestra en la figura 1. Esta primera parte se corresponde con la observación, en la que emplea la cinemática de las huellas para establecer la división de la secuencia en tramos. Esta heurística fue sugerida por la profesora oralmente.

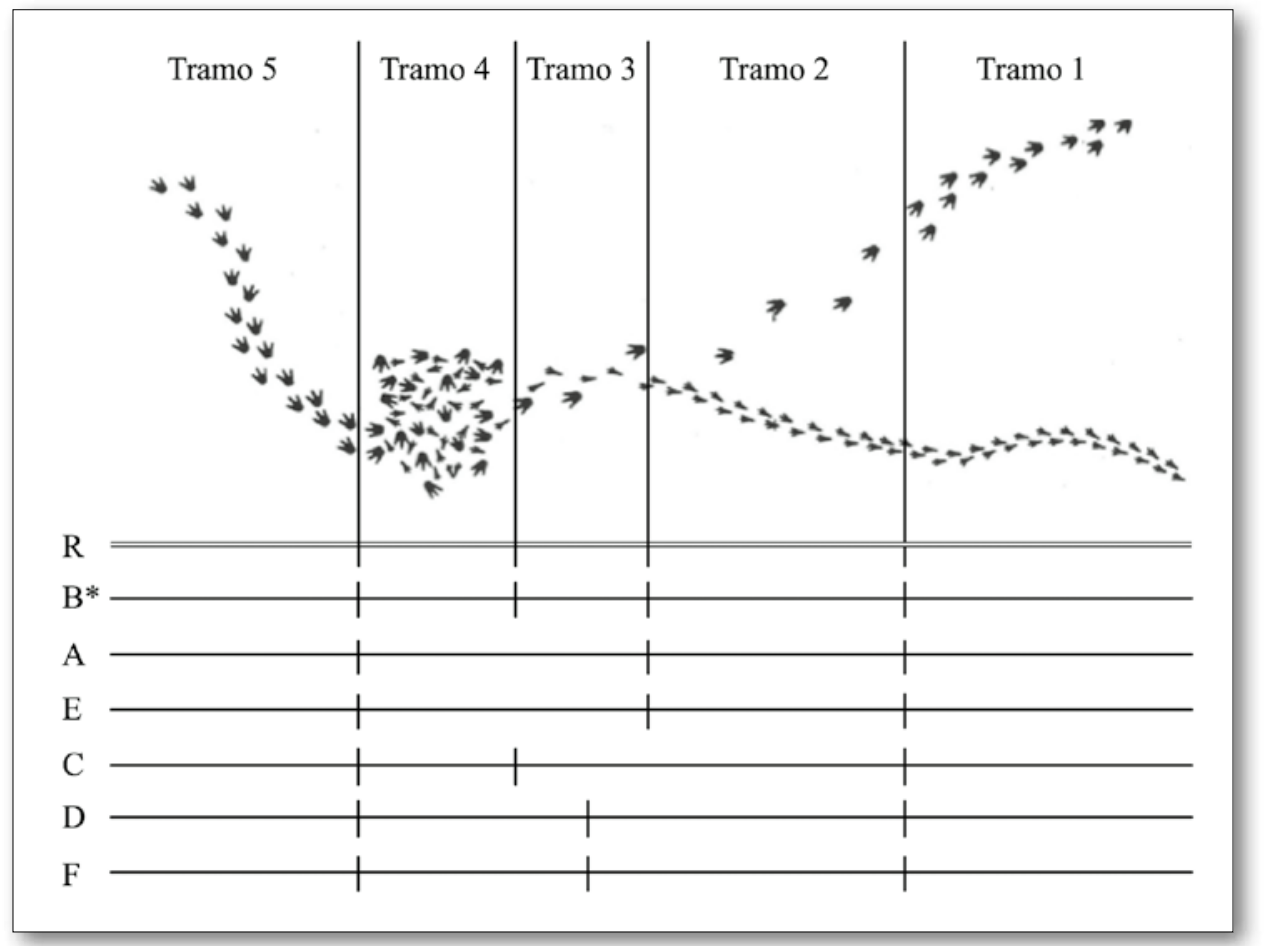

Fig. 1. Comparación de la división de la secuencia de pisadas del referencial (R) con cada grupo (A, B, C, D, E, F).

El grupo realizó la separación de los tramos igual al $\mathrm{R}$, pero fue en los últimos minutos de la sesión y durante la puesta en común.

A grandes rasgos, se encontró que todos los grupos identificaron los tramos 1 y 5 , mientras que no todos los tramos intermedios fueron establecidos, por lo que entendemos que no consiguieron toda la información disponible. Sorprendentemente, el único grupo cuya división en tramos coincide con nuestro referente es el grupo $B$, pese a que sus integrantes apenas se implicaron en la tarea. Los grupos A y E son bastante similares al referencial, salvo que fusionan los tramos 3 y 4 , lo que entendemos que es una interpretación de que la persecución y pelea es incluida en un mismo episodio. Sin embargo, el grupo $\mathrm{C}$ une los tramos 2 y 3 en uno, caracterizado por que "ambos corren". Finalmente, los grupos más distantes con el referencial son el $\mathrm{D}$ y el F, ya que solo establecen tres partes. Estos grupos solo se centran en buscar explicaciones a los tramos 4 y 5 . 
Las observaciones realizadas por los estudiantes les permitieron extraer la información contenida en esas huellas, para luego poder emplearla en sus justificaciones. Además, resultó interesante para ver cómo hubo una ligera unanimidad de opiniones acerca de lo que se observa y los datos que obtienen. No obstante, en las inferencias hay una variada gama de explicaciones, lo que nos afirma en la validez de la propuesta.

Las inferencias que realizó el alumnado también se analizan por tramos. Así, comprobamos que lo que ocurre en los primeros tramos de la secuencia de icnitas no presenta mayor complejidad, por lo que es interpretado por los distintos grupos de estudiantes de la misma forma: primero van andando, a continuación uno comienza a correr y el otro escapa, pero ¿qué ocurre en las huellas entremezcladas para que después solo salga un individuo? Para dar respuesta a esta cuestión deben ser interpretados los dos últimos tramos, el 4 y el 5 . Al hecho de que en ellos es posible formular un mayor número de respuestas es a lo que los grupos dedican mayor parte de la sesión. Para estos últimos tramos las inferencias son muy diversas, como puede apreciarse en los siguientes ejemplos:

Ángela (L.4.1): "Yo creo que se pelearon, mira, se pelearon, murió uno [tramo 4] y siguió el otro [tramo 5]".

Carlos (L.107): “...va por aquí, por aquí, tiki tiki tiki, y aquí se gira, es como decía yo, se gira para pelear contra él. El otro llega, por aquí, por aquí, por aquí, y ve que el otro está delante y empiezan a pelearse, empiezan a dar vueltas, lo mata por aquí, que es donde están las dos huellas juntas [tramo 4]”.

David (L.28, puesta común): “También se podía pelear por el territorio el de las pisadas pequeñas [tramo 4]”.

David (L.50, puesta común): "Profe, pero este puede volar, y a lo mejor desaparece de ahí. Pilló vuelo [tramo 4]".

Aunque la división de la secuencia de huellas sea más o menos adecuada, la atención que prestan a cada uno de los tramos depende de las inferencias que hacen. Consecuentemente, su discurso no es lineal, puesto que sus intervenciones se mueven de unos tramos a otros a medida que producen inferencias sobre lo ocurrido. A continuación, se muestra la progresión del discurso para cada tramo a lo largo de la sesión (figuras $2 \mathrm{~A}$ y $2 \mathrm{~B}$ ); como puede verse en las líneas temporales, sin escala, de los grupos A y D.

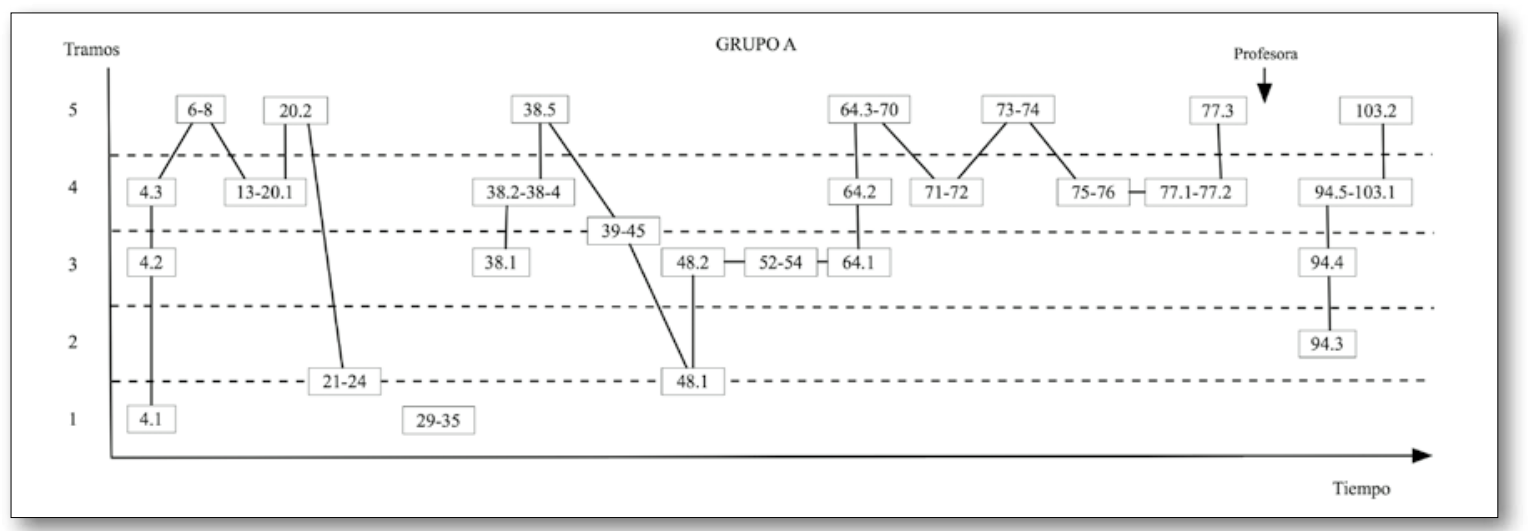

Fig. 2A. Línea temporal de las intervenciones del grupo A.

Nota: Las líneas continuas, que unen los turnos, muestran las intervenciones sucesivas centradas en los distintos tramos de la secuencia de icnitas en que los estudiantes focalizan su atención. Los números indican los turnos de los estudiantes. 


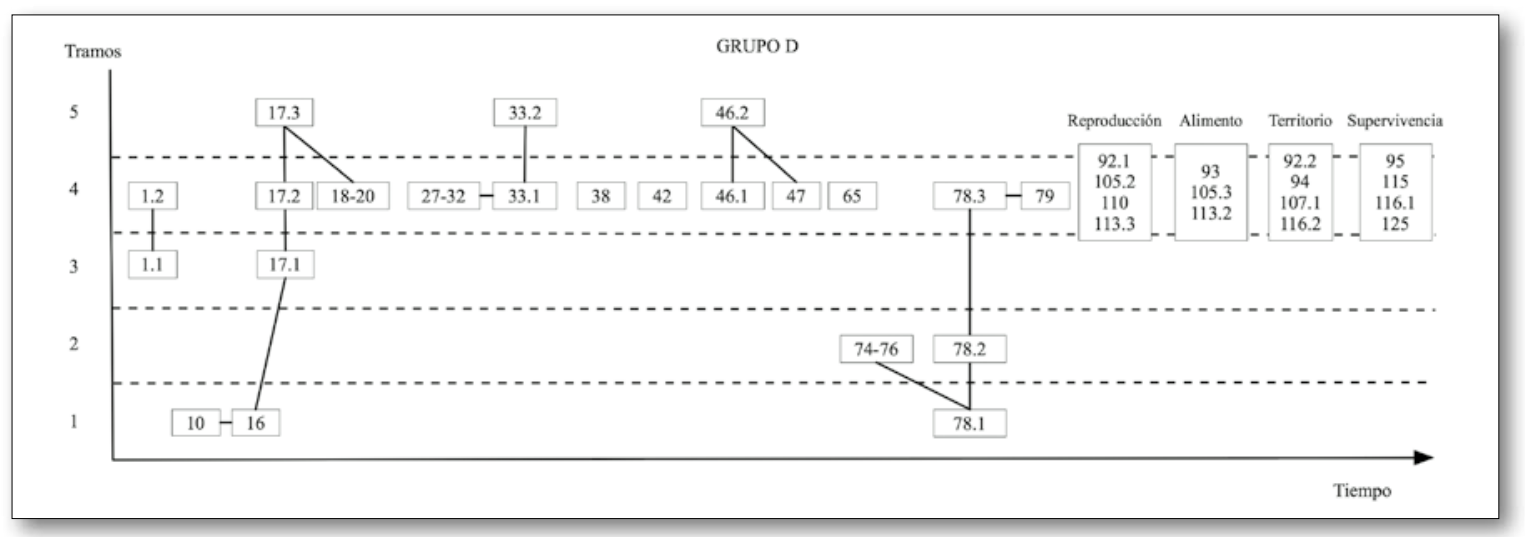

Fig. 2B. Línea temporal de las intervenciones del grupo D.

Nota: Las líneas continuas, que unen los turnos, muestran las intervenciones sucesivas centradas en los distintos tramos de la secuencia de icnitas en que los estudiantes focalizan su atención. Los números indican los turnos de los estudiantes.

En estas dos gráficas se observan unas pautas generales: tras la presentación de la actividad, durante unos 3-4 minutos, abordaron el conjunto de la secuencia estableciendo la inferencia principal (grupo A, líneas 4-8; grupo D, líneas 10-17.3) de lo que ocurrió y, a partir de ahí, valoraron diferentes alternativas. Todos los grupos coinciden en dedicar más tiempo a la interpretación de los tramos 4 y 5 .

Como se puede apreciar en la gráfica del grupo A (figura 2A), destaca la intervención de la profesora solicitando una síntesis de sus inferencias. Sin embargo, el grupo D (figura 2B) no hace apenas análisis longitudinales, sino que únicamente se centra en dar posibles soluciones a los tramos 4 y 5 , pero sin justificarlos, como se verá en el análisis de sus argumentos.

\section{Análisis de la construcción del conocimiento a través de la argumentación}

La argumentación de los pequeños grupos se ha representado siguiendo el modelo de argumentación de Toulmin (1958), en el que se encuentran reflejadas las observaciones, que se sitúan en el nivel de los datos, las inferencias, que son las justificaciones, y, por último, las conclusiones. Es decir, ha sido un proceso de obtención de datos a través de la observación e inferencia que se han transformado en pruebas con las que justificar sus argumentos.

El segundo objetivo propuesto corresponde, como se indicó, al análisis del discurso argumentativo mediante el cual los estudiantes justifican sus conclusiones, que se desarrolla a continuación.

Se comienza con el perfil detallado de los argumentos de los grupos A y D, para finalmente analizar las pruebas y los argumentos empleados durante la puesta en común de todos los grupos. Cabe señalar que los argumentos fueron esquematizados por los autores a partir de las transcripciones.

El argumento principal del grupo A posee cierta sofisticación al estar sustentado por 5 subargumentos, una justificación con respaldo y con una refutación. Otra característica importante es que todos los integrantes toman parte en la construcción del argumento, contrastan sus ideas y formulan una síntesis final (esquema 2). 


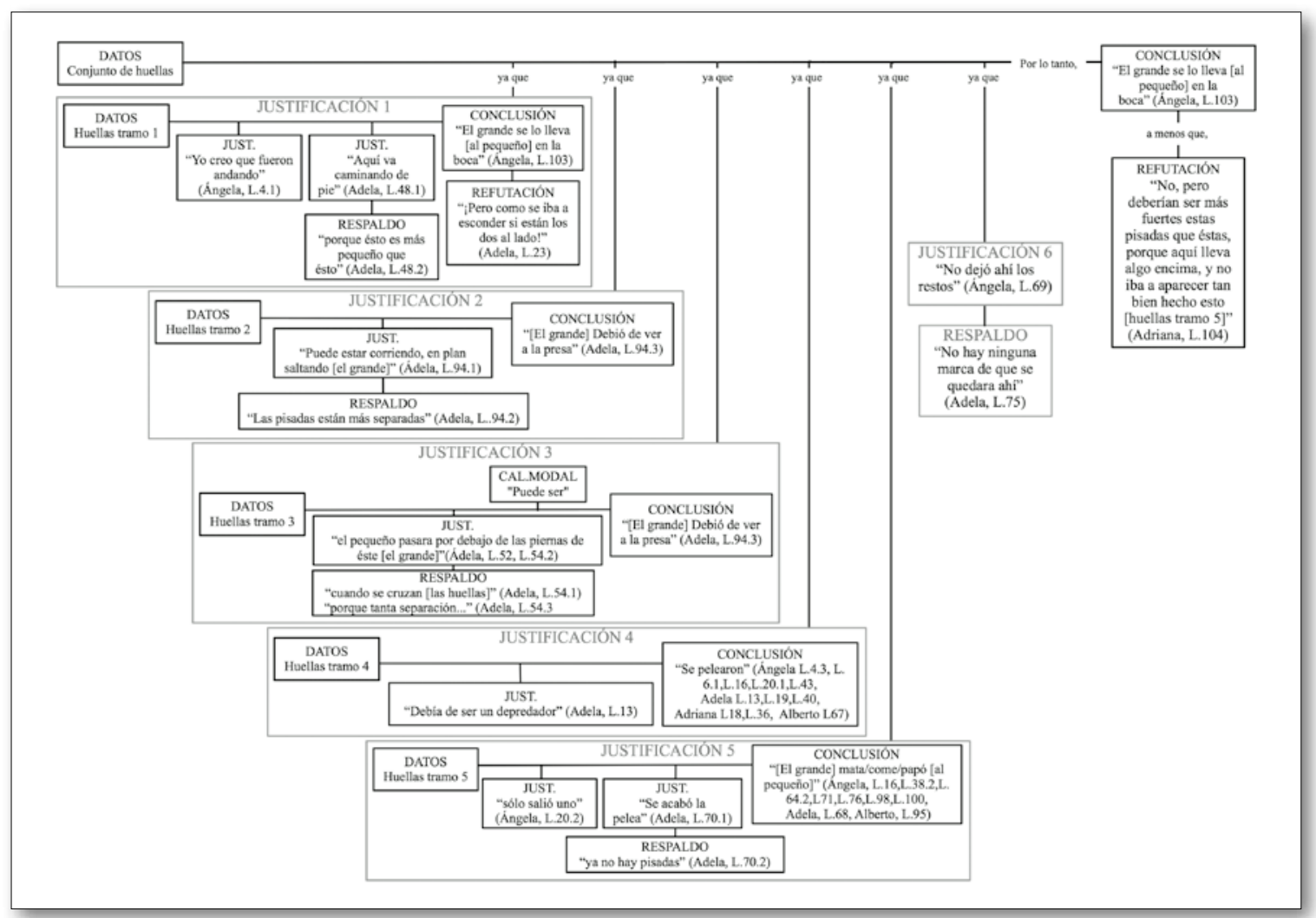

Esquema 2. Argumentación del grupo A

La refutación que formula Adriana tiene forma de argumento, debido a que en su afirmación emplea los datos que proporciona la imagen para justificar su contraposición ante el hecho de que "el grande se llevara en la boca al pequeño” (Ángela, L.103):

Adriana (L.104): "No, pero deberían ser más fuertes estas pisadas que estas, porque aquí lleva algo encima, y no iba a aparecer tan bien hecho".

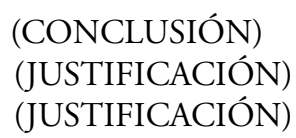

La complejidad del argumento del grupo A radica en el gran número de respaldos que emplean para sustentar las justificaciones; un buen ejemplo de ello se da durante la intervención de Adela:

Adela (L.94): "Pueden estar corriendo, en plan saltando.

Por eso las pisadas están más separadas”.

(JUSTIFICACIÓN)

(RESPALDO)

Este respaldo constituye una de las principales diferencias de calidad de argumento entre los grupos, puesto que no se trata de una simple opinión, sino que muestra el dato que respalda su interpretación.

En el momento de descifrar los tramos 4 y 5 conjuntamente, Adela enuncia un contraargumento (esquema 3) como alternativa al argumento principal, en el que se considera al individuo pequeño un pájaro y que saliese volando; sin embargo, esta alternativa no es aceptada por el resto del grupo y queda solo en una propuesta. 


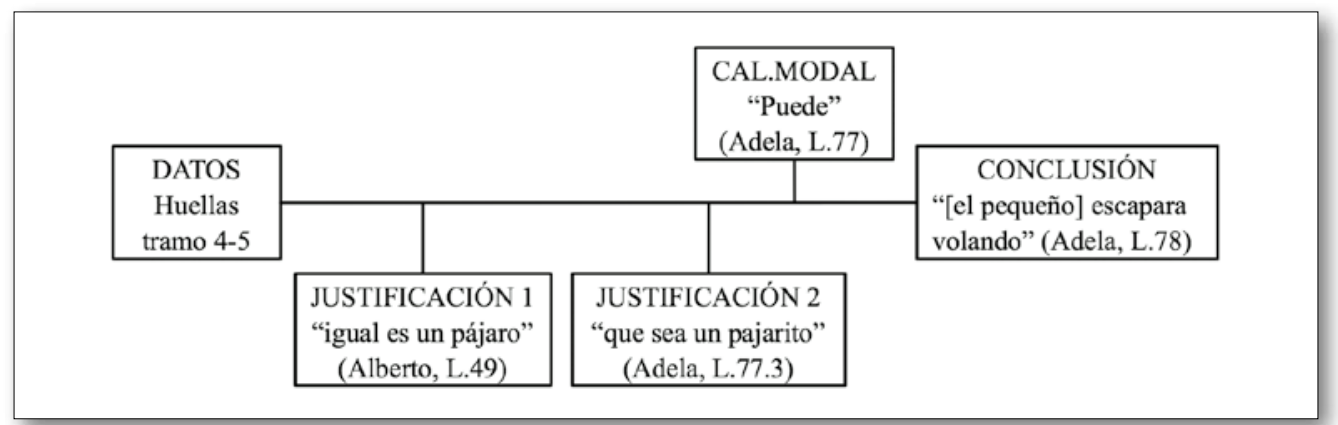

Esquema 3. Grupo A, donde justifican la posibilidad de que sea un pájaro

En el caso del grupo D, sus integrantes realizaron varios argumentos que explican la secuencia de huellas, de los que mostramos el más elaborado, el esquema 4. En este argumento enuncian 4 subargumentos; falta el del tramo 3, como consecuencia de que no lo identificaron durante la observación de las huellas. Además, apenas detallan las inferencias con las que apoyar las conclusiones a las que llegan. El hecho de que no realicen una correcta observación de las huellas, ni las correspondientes inferencias, es lo que los lleva a enumerar varias conclusiones sin decantarse por ninguna de ellas.

La particularidad de este grupo, compartida con el grupo F, es la ausencia de un argumento debidamente justificado y sustentado en los datos proporcionados. Durante el debate en pequeño grupo, exponen conclusiones diferentes para los tramos 4 y 5, como se detalló en la figura 2B, que son: pelea por el territorio, pelea por el alimento, pelea por la supervivencia y reproducción. Ellos denominan sus conclusiones como "hipótesis"; sin embargo, consideramos apropiado emplear el término conclusión, al enmarcarse en un proceso argumentativo.

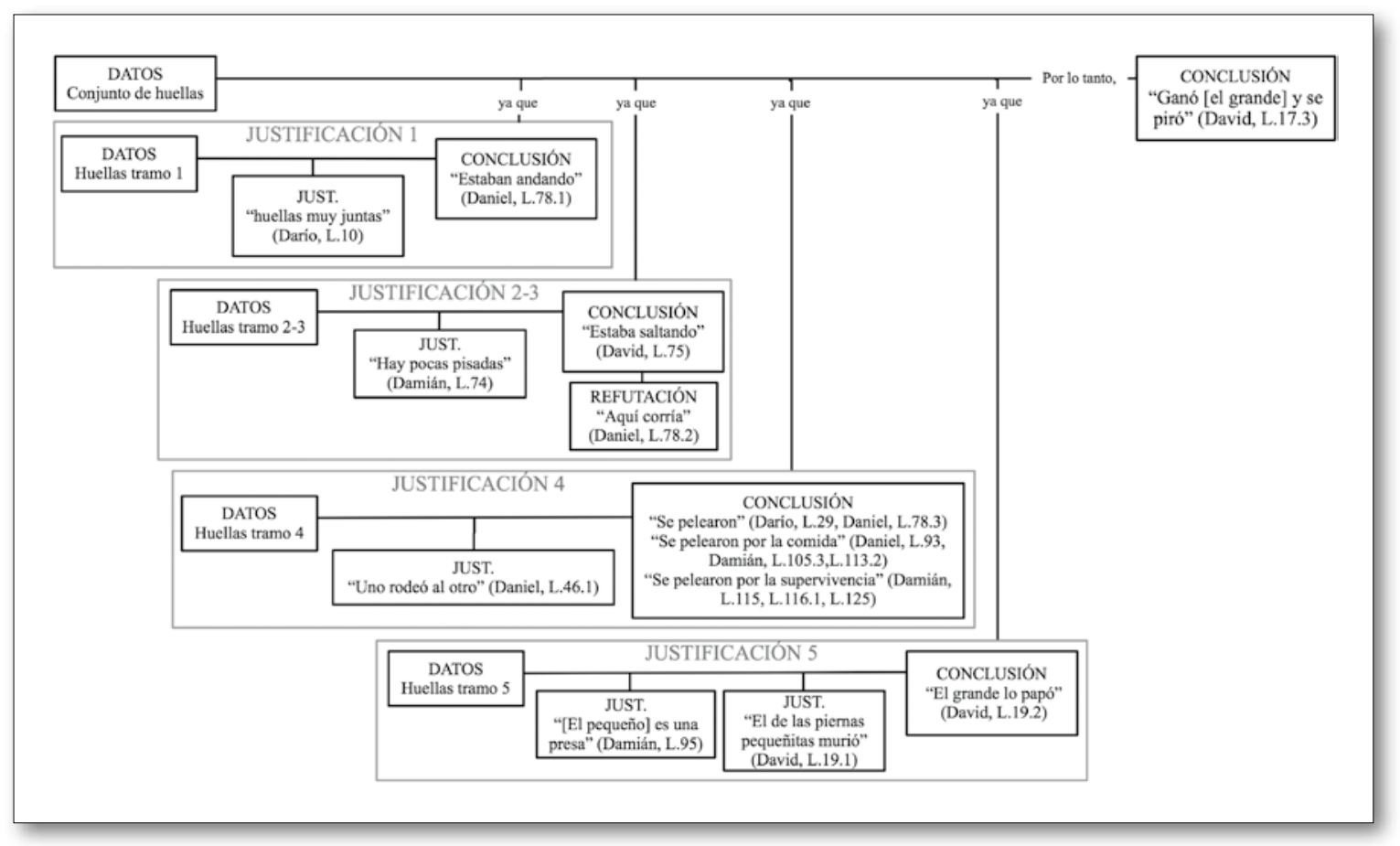

Esquema 4. Argumentación del grupo D 
En el diálogo entre los cuatro chicos del grupo D hay una ausencia total de intento de persuasión entre ellos, centrándose la mayor parte del tiempo en enunciar las diferentes interpretaciones sin concretar ninguna. Como consecuencia, no se formulan refutaciones ni respaldos; esto puede ser un indicador de que no captaron el objetivo del problema, que consistía en establecer una conclusión.

Para concluir con los argumentos de los grupos, comprobamos que la co-construcción de los argumentos dentro de cada grupo corrobora la opinión de Kuhn (2005), quien afirma que los estudiantes realizan más progresos y más rápidos cuando trabajan en grupos en lugar de individualmente, lo cual se pone de manifiesto con la cantidad de inferencias establecidas en los grupos a partir de los datos aportados por la actividad.

La puesta en común, que se aborda a continuación, enriqueció la actividad argumentativa al sentir los estudiantes la necesidad de reforzar sus conclusiones con una mayor aportación de pruebas. En la tabla 1 se muestra la sucesión de las conclusiones de los argumentos establecidos por cada grupo.

En la intervención de Eliseo, portavoz del grupo E, se expone la primera conclusión de la puesta en común. Eliseo (L.4) propone que "hubo una pelea que ganó el depredador y este se comió a la presa" (conclusión 1). Nuestra opinión acerca de este argumento es que está debidamente justificado y engloba todos los tramos, sin embargo, no hace uso de ningún respaldo que apoye las afirmaciones de cada tramo, es decir, no detalla las observaciones que los condujeron a ellas. Para el grupo E, este argumento resuelve el problema por completo, ya que según Ernesto (L.76) "se comieron hasta los huesos".

Tabla 1.

Sucesión de los argumentos durante la puesta en común

\begin{tabular}{|c|c|c|c|}
\hline Grupo & N.o & Argumento & Refutación \\
\hline $\mathrm{E}$ & 1 & $\begin{array}{l}\text { "Hubo una pelea que ganó el depredador y este se } \\
\text { comió a la presa". } \\
\text { "Se comieron hasta los huesos". }\end{array}$ & \\
\hline $\mathrm{C}$ & 2 & "El depredador mató a la presa". & \\
\hline \multirow{6}{*}{$\mathrm{D}$} & \multirow{3}{*}{3} & \multirow{3}{*}{$\begin{array}{l}\text { "Podía pelear por el territorio el de las pisadas pe- } \\
\text { queñas". }\end{array}$} & 1. Eliseo: "Escapa, ‘’andando? [tono irónico]". \\
\hline & & & 2. Eloy: "Y el otro queda ahí para siempre". \\
\hline & & & $\begin{array}{l}\text { 3. Carlos: “伊or qué del de las pisadas pequeñas no } \\
\text { se ven más huellas de él en otro sitio?”. }\end{array}$ \\
\hline & \multirow[t]{2}{*}{4} & \multirow[t]{2}{*}{ "Murieron los dos". } & $\begin{array}{l}\text { 1. Félix: "Sí, murieron y uno se fue andando [con } \\
\text { ironía]". }\end{array}$ \\
\hline & & & 2. Eloy: "Si muriese ahí uno debería haber fósiles". \\
\hline & 5 & “[El pequeño] pilló vuelo". & \\
\hline
\end{tabular}

A continuación, Celso (L.6) expone la conclusión de su grupo: "El depredador mató a la presa" (conclusión 2), semejante a la expuesta por Eliseo.

El siguiente en intervenir es David (L.28), del grupo D, que expone una de sus tres conclusiones: "Podía pelear por el territorio el de las pisadas pequeñas" (conclusión 3).

Esta conclusión (3) proporciona una perspectiva completamente contraria a las anteriores, por el hecho de considerar que el grande no es el que gana la pelea, sino quien la pierde. Este argumento proporciona una explicación a la secuencia de pisadas que en un primer momento podría parecer válido pero los compañeros hacen sucesivas refutaciones a las justificaciones:

Refutación 1. Eliseo (L.40): "Escapa, ¿̨andando? [tono irónico]”.

Refutación 2. Eloy (L.43): "Y el otro queda ahí para siempre [las huellas no salen del entramado]".

Refutación 3. Carlos (L.75):“¿Por qué del de las pisadas pequeñas no se ven más huellas de él en otro sitio?”. 
La intervención de Carlos se enuncia a modo de pregunta, pero su intención es contradecir a David, por lo tanto se puede considerar una refutación en términos de Kuhn (1991), puesto que se opone a la justificación de David, y además entraña otro argumento, en el cual hay una conclusión: "el pequeño no se quedó en el territorio", y una justificación: "no hay más pisadas del pequeño en otro sitio".

Ante estas refutaciones, David decide enunciar una segunda conclusión, que a su vez constituye un contraargumento del suyo anterior (L.44): "murieron los dos" (conclusión 4). Pero nuevamente, comienzan las refutaciones:

Refutación 1. Félix (L.45): "Sí, murieron y uno se fue andando [con ironía]”.

Refutación 2. Eloy (L.46): "Si muriese ahí uno, debería haber fósiles".

Esta última refutación de Eloy, a nuestro parecer, es una afirmación clave para la resolución del problema, debido a que si quedasen huesos fósiles registrados, las posibles alternativas quedarían reducidas a una: el grande mató al pequeño y ni siquiera sería necesario que se lo comiese.

Ambas refutaciones van dirigidas a la conclusión de David y, en los dos casos, presentan una justificación apoyada en las pruebas obtenidas de la imagen, con lo cual demuestran lo insensato que resulta ese contraargumento (conclusión 4).

La tercera y última conclusión del grupo D es la que David (L.50) formula: "(el pequeño) pilló vuelo" (conclusión 5), lo que parece convencer al resto de los grupos.

Lo interesante de todas las refutaciones y contraargumentos ya descritos es que constituyen los eslabones finales de la argumentación, lo que nos da idea de la gran complejidad y calidad del discurso. Se formularon contraargumentos de ambos tipos, ya que la ausencia de datos aumenta la inestabilidad de los argumentos propuestos, lo que desembocó en que se debatiese la opinión de los compañeros, e incluso la propia, con contraargumentos. Si atendemos a las refutaciones, se emplearon sobre todo en la puesta en común.

Siguiendo la opinión de Kuhn (1991), el uso de ambas figuras pone de manifiesto que los individuos interiorizaron el argumento del oponente para poder proponer pruebas o afirmaciones en contra. Además, se comprueba que cuando hubo ausencia de contraargumentos o refutaciones las afirmaciones no necesitaron de pruebas (Kuhn, 2005).

A fin de proporcionar un resumen del proceso de elaboración de conclusiones para esta secuencia de icnitas se elaboró la tabla 2 , en la que se muestran todas las observaciones, inferencias y conclusiones realizadas en el aula.

Tabla 2.

Resumen de las observaciones e inferencias explícitas que generaron las conclusiones

\begin{tabular}{|c|c|c|c|}
\hline & OBSERVACIÓN & INFERENCIA & CONCLUSIÓN \\
\hline TRAMO 1 & Huellas más juntas & Van caminando & \\
\hline TRAMO 2 & Huellas más separadas & Van corriendo & $\begin{array}{l}\text { El grande se quiere comer al pequeño } \\
\text { El pequeńo escapa }\end{array}$ \\
\hline TRAMO 3 & & Se mueven en círculos & Lucha \\
\hline TRAMO 4 & $\begin{array}{l}\text { Huellas grandes } \\
\text { Huellas pequeñas }\end{array}$ & $\begin{array}{l}\text { El animal de mayor tamaño es de- } \\
\text { predador } \\
\text { El animal pequeño es una presa y el } \\
\text { grande es un depredador }\end{array}$ & El grande se comió al pequeño \\
\hline TRAMO 5 & $\begin{array}{l}\text { Solo quedan unas huellas } \\
\text { No quedan restos fósiles del } \\
\text { pequeño }\end{array}$ & El pequeño es un pájaro & $\begin{array}{l}\text { El pequeño salió volando } \\
\text { El grande lleva al pequeño en la boca } \\
\text { El grande lleva al pequeño encima }\end{array}$ \\
\hline
\end{tabular}




\section{CONCLUSIONES}

A la luz de los resultados, se extraen las siguientes conclusiones vinculadas a los objetivos de la investigación propuestos al comienzo.

En primer lugar, se puede afirmar que la interpretación del alumnado sobre la secuencia de icnitas resultó adecuada, aunque desordenada. La resolución de este problema requería establecer una planificación, la cual los llevaría a realizar inferencias de forma secuencial. Sin embargo, tras presentar la actividad, los estudiantes leyeron el enunciado y emitieron sus primeras conclusiones de forma desordenada (figuras $2 \mathrm{~A}$ y $2 \mathrm{~B}$ ), hasta que la profesora intervino indicándoles que deberían realizar las inferencias de forma ordenada, es decir, dividir la secuencia en tramos. A partir de ahí es cuando se detienen a observar con detalle las huellas y comienzan a realizar inferencias con las cuales apoyan sus conclusiones. Con esto se demuestra la necesidad de estimular al alumnado a que razone las acciones que realiza y las que realizará (Sanmartí, 2002), a fin de que mejore en la resolución de un problema.

En segundo lugar, el contexto de la actividad contribuyó a que los estudiantes realizasen diversas inferencias en la interpretación de la secuencia de icnitas, lo que derivó en un debate argumentativo acerca de qué conclusión era la más adecuada. Durante este debate, los estudiantes emplearon argumentos bien elaborados y se produjeron refutaciones, por lo que consideramos que esta actividad promueve una argumentación de alta calidad en el sentido indicado por Erduran, Simon y Osborne (2004).

Es importante también considerar el papel de la profesora durante el desarrollo de la actividad, al fomentar ambientes que promuevan la argumentación y al demandar pruebas a los estudiantes para que estos justifiquen sus afirmaciones, promoviendo además la reflexión y el metaconocimiento, en concordancia con lo indicado por Jiménez-Aleixandre (2008).

En definitiva, la puesta en práctica de este tipo de actividades permite a los alumnos actuar como productores de conocimiento (Jiménez y Pereiro, 2002). Además, como indica Del Carmen (2011), es importante que los alumnos tengan la oportunidad de enfrentarse a la resolución de un problema científico con todo lo que ello conlleva, es decir, un intento racional de dar respuesta argumentada y con suficientes pruebas a una pregunta o resolver un problema.

Para finalizar, como implicación educativa más relevante, comprobamos que con esta forma de trabajo los estudiantes desarrollan su capacidad argumentativa. Saber argumentar es la habilidad que permitirá a los alumnos buscar y adquirir conocimiento y emplearlo para hacer juicios (Kuhn, 2005) sobre los nuevos avances científicos en los que la sociedad se ve inmersa.

\section{AGRADECIMIENTOS}

$\mathrm{Al}$ alumnado, por su participación e implicación en la propuesta didáctica.

Este estudio se desarrolla en el marco del proyecto EDU-2012-38022-C02-01, financiado por el Ministerio de Economía y Competitividad.

\section{REFERENCIAS BIBLIOGRÁFICAS}

Anguita Virella, F. y Moreno Serrano, F. (1978). GEOlOGía: Procesos internos. Zaragoza: Edelvives.

Bachelard, G. (1938). La formation de l'esprit scientifique. París: Vrin [Traducción: La formación del espíritu científico, 1974, Buenos Aires: Siglo XXI].

Cañas, A.; Martín-Díaz, M. J. y NiedA, J. (2007). La competencia en el conocimiento y la interacción con el mundo fisico. La competencia cientifica. Madrid: Alianza. 
Carmen, L. del (2011). El lugar de los trabajos prácticos en la construcción del conocimiento científico en la enseñanza de la Biología y la Geología. En: P. Cañal (coord.). Didáctica de la Biología y Geología. Madrid: Graó.

Chinn, C. A. y Malhotra, B. A. (2002) Epistemologically authentic inquiry in schools: A theoretical framework for evaluating inquiry tasks. Science Education, 86(2), pp. 175-218. http://dx.doi.org/10.1002/sce.10001

Díaz De Bustamante, J. y Jiménez Aleixandre, M. P. (1999). Aprender ciencias, hacer ciencias: resolver problemas en clase. Alambique. Didáctica de las ciencias experimentales, 20, pp. 9-16.

Driver, R.; Newton, P. y Osborne, J. (2000). Establishing the norms of scientific argumentation in classrooms. Science Education, 84, pp. 287-312. http://dx.doi.org/10.1002/(SICI) 1098-237X(200005)84:3<287::AID-SCE1>3.0.CO;2-A

Duschl, R. y Erduran, S. (1996). Modelling the growth of scientific knowledge. En G. Weldorf; J. Osborne y P. Scott. Research in science education in Europe. Londres: Farmer Press.

Erduran, S.; Simon, S. y Osborne, J. (2004). TAPping into argumentation: Developments in the application of Toulmin's argument pattern for studying science discourse. Science Education, 88(6), pp. 915-933.

http://dx.doi.org/10.1002/sce.20012

Hurd, D.; Johnson, S. M.; Matthias, G. F.; Mclaughlin, C. W.; Snyder, E. B. y Wright, J. D. (1989). General Science: A voyage of Discover. New Jersey: Prentice Hall.

IzQuierdo, M. (2000). Fundamentos epistemológicos. En F. J. Perales Palacios y P. Cañal de León. Didáctica de las Ciencias Experimentales. Alcoy: Marfil.

Jiménez Aleixandre, M. P. (1996). Dubidar para aprender. Vigo: Xerais.

Jiménez Aleixandre, M. P. (1998). Diseño curricular: indagación y razonamiento con el lenguaje de las ciencias. Enseñanza de las ciencias, 16(2), pp. 203-216.

Jiménez Aleixandre, M. P. (2008). Designing Argumentation Learning Environments. En S. Erduran y M. P. Jiménez-Aleixandre (eds.). Argumentation in science education. Dordrecht: Springer.

Jiménez Aleixandre, M. P. (2010). 10 ideas clave: Competencias en argumentación y uso de pruebas. Barcelona: Graó.

Jiménez Aleixandre, M. P. (2011). Argumentación y uso de pruebas: construcción, evaluación y comunicación de explicaciones en Biología y Geología. En P. Cañal (coord.). Didáctica de la Biología y Geología. Madrid: Graó.

Jiménez Aleixandre, M. P.; Bravo, B. y Puig, B. (2009). ¿Cómo aprende el alumnado a evaluar pruebas? Aula de Innovación Educativa, 186, pp. 10-12.

Jiménez Aleixandre, M. P. y Pereiro Muñoz, C. (2002). Knowledge producers or knowledge consumers? Argumentation and decision making about environmental management. International Journal of Science Education, 24, pp. 1171-1190. http://dx.doi.org/10.1080/09500690210134857

Kelly, G. J. y TaKaO, A. (2002). Epistemic levels in argument: An analysis of university oceanography students' use of evidence in writing. Science Education, 86, pp. 314-342. http://dx.doi.org/10.1002/sce.10024

Kunn, D. (1991). The skills of argument. Cambridge: Cambridge University Press. http://dx.doi.org/10.1017/CBO9780511571350

Kunn, D. (2005). Education for thinking. Harvard: Harvard University Press

Kunn, T. S. (1962). La estructura de las revoluciones cientificas. México DF: Fondo de Cultura Económica 
Lederman, N. G. y Abd-El-Khalick, F. (1998). Avoiding De-Natured Science: Activities that promote understandings of the Nature of Science. En W. F. McComas (ed.). The nature of science in science education. Dordrecht: Kluwer, pp. 83-26

Lockley, M. G. (1993). Siguiendo las huellas de los dinosaurios. Madrid: McGraw-Hill

OCDE (2006). PISA 2006. Marco de la evaluación: conocimientos y habilidades en Ciencias, Matemáticas y Lectura. Madrid: Santillana. Ministerio de Educación y Ciencia. Disponible en: <http://www. oecd.org>. (Última consulta: 1 de julio de 2011).

Pedrinaci, E. (2001). Los procesos geológicos internos. Madrid: Síntesis Educación.

Sanmartí, N. (2002). Didáctica de las ciencias en la educación secundaria obligatoria. Madrid: Síntesis Educación.

Sequeiros, L.; Pedrinaci, E. y Berjillos, P. (1996). Cómo enseñar y aprender los significados del tiempo geológico: algunos ejemplos. Enseñanza de las Ciencias de la Tierra, 4(2), pp. 113-119.

Toulmin, S. (1958). The uses of argument. Nueva York: Cambridge University Press. 


\section{ANEXO \\ Actividad didáctica propuesta}

Los alumnos de primero de bachillerato del IEs Antonio Machado de Soria encontraron un conjunto de pisadas en un yacimiento paleontológico próximo a la ciudad. Tras tomarles fotografías y analizarlas, no lograron esclarecer qué ocurrió en esa secuencia de pisadas. Por este motivo decidieron enviar un dibujo de estas huellas a diferentes IEs de España para que otros/as alumnos/as los ayudaran. Este conjunto de pisadas es el que se muestra a continuación:

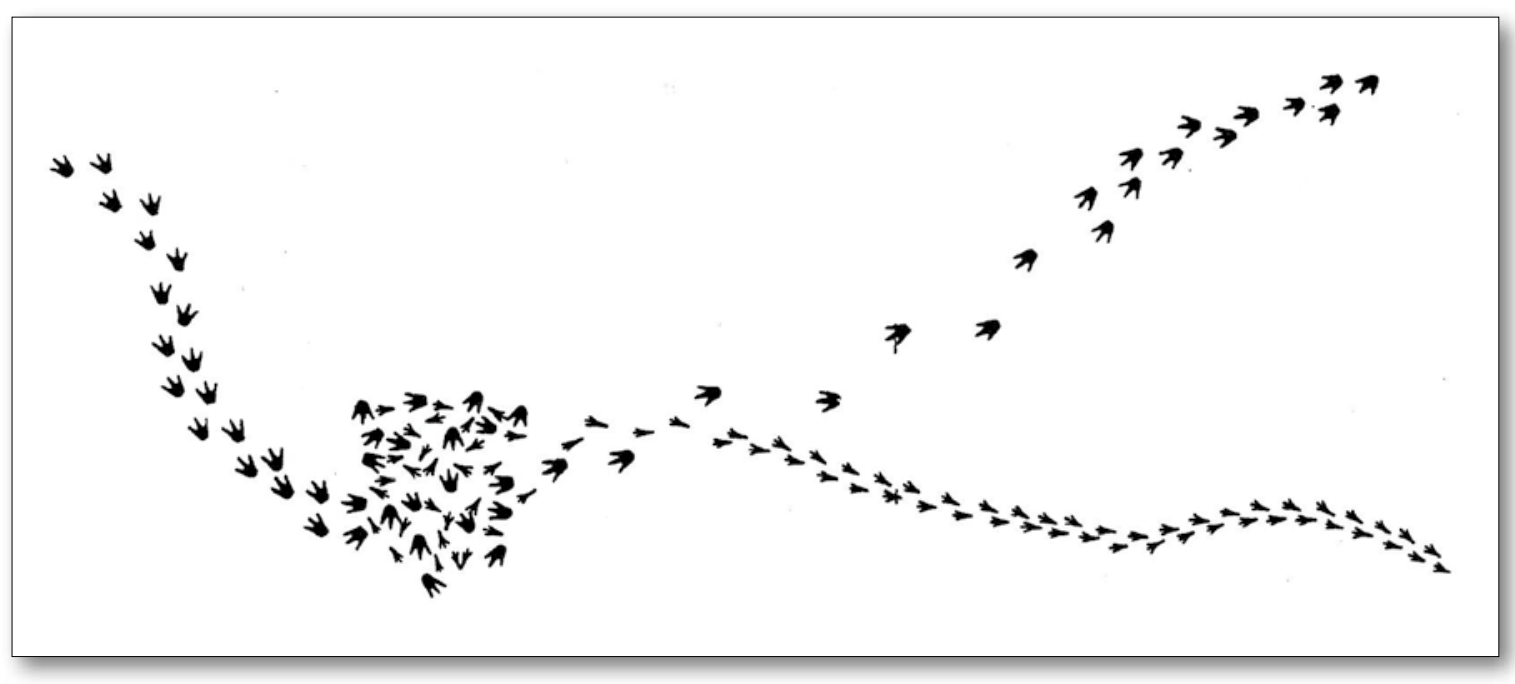

Por grupos, debéis esclarecer qué ocurrió en esa secuencia de pisadas. Después, todos los miembros del aula deberán extraer una conclusión, que será la que se envíe como respuesta a los compañeros del IES de Soria. ¿Necesitaríais conocer alguna otra cosa? 


\title{
Argumentation and use of evidence: making inferences about a sequence of footprints
}

\author{
Paloma Blanco Anaya, Joaquín Díaz de Bustamante \\ Departamento de Didáctica das Ciencias Experimentais \\ Universidade de Santiago de Compostela \\ paloma.blanco@usc.es,joaquin.diaz@usc.es
}

The aim of this study is twofold: 1) to promote students' scientific reasoning through observation, making inferences and posing hypotheses or drawing conclusions, and 2) to analyse their argumentative discourse to justify their conclusions.

The task is designed as an authentic problem set on Soria's paleontological bed (Spain). The context presented with the task is that some students have found a set of fossil footprints but they aren't able to ascertain what happened there. So these students decided to ask for help sending a picture of the tracks to other schools to be interpreted by other students. Although the footprints were taken from Lederman and Abd-El-Khalick (1998), the original source is Hurd et al. (1989). Lockley (1993) asserts that, although it could seem like a real sequence of footprints to non-experts, it was designed as a task for pupils.

It is important to point out that it is not a geology problem itself, because its main purpose is to develop students' scientific competence. In other words, taking into account the three dimensions of scientific competence proposed in PISA (OCDE, 2006), this problem contributes to explain scientific phenomena, when students have to describe and interpret the footprints, and to use scientific evidence, to develop arguments and persuade their classmates. According to this approach, authentic problems provide a wide range of possible solutions and students must choose the most appropriate, using their argumentative skills.

The activity was implemented in a classroom of 11 th grade students $(\mathrm{N}=23)$, who were enrolled in "Ciencias para el mundo contemporáneo". The whole class was divided into four groups, and each one was recorded on audio and video, to be then transcribed.

On a first level of analysis, we examine the stages in which each group divided the footprints sequence so as to recognise the inferences made on them. On a second level of analysis, we interpret students' argumentation using the Toulmin's argumentative pattern (1958), modified by Kelly and Takao's pattern (2002).

Regarding the first research objective, students' interpretation of the footprints sequence was adequate but disorganised. Solving this problem required them to establish a schedule, which would lead them to make inferences sequentially. However, after showing them the activity, students read the statement and proposed their first findings in a disorganised way until the teacher told them that they should make inferences orderly, that is, dividing the sequence into sections. From that moment on, they observed the tracks in detail and began to make inferences to support their conclusions.

Considering the second research objective, it has been found that all groups elaborate arguments to justify their tracks interpretation. Nevertheless, the best arguments took place in those groups whose students showed disagreement with each other, as this entailed that they had to use more evidence to enrich their justifications. Once the groups drew their conclusions, they shared them with the whole class, trying to select the best conclusion to be sent to Soria's school. During this discussion, students used well-prepared arguments and rebuttals; therefore, we consider that this activity promotes a high quality argumentation, following Erduran, Simon and Osborne (2004).

To conclude, the implementation of these activities allows students to act as producers of knowledge (Jiménez and Pereiro, 2002) and also, as del Carmen (2011) pointed out, it is important that students have the opportunity to face scientific problem solving with everything that it entails, that is, a reasoned attempt to provide sufficient evidence to answer a question or solve a problem. 